\title{
Clustering Based Space-Time Network Coding
}

\author{
Wei Guan, and K. J. Ray Liu \\ Department of Electrical and Computer Engineering \\ University of Maryland, College Park, MD 20742 \\ Email: \{wguan, kjrliu\}@umd.edu
}

\begin{abstract}
Many-to-one communication is a challenging problem in practice due to channel fading and multi-user interferences. In this work, a new protocol that leverages spatial diversity through space-time network coding is proposed. The $N$ source nodes are first divided into $K$ clusters, each having $Q$ nodes, and the clusters send data successively in a time-division multiple access way. Each node behaves as a decode-and-forward relay to other clusters, and uses linear coding to combine the local symbol and the relayed symbols. To separate the multi-source signals, each node has a unique signature waveform, and linear decorrelator is used at the receivers. Both the exact Symbol Error Rate (SER) and the asymptotic SER at high signal-tonoise ratios of the $M$-ary phase-shift keying signal are studied then. It is shown that a diversity order of $(N-Q+1)$ can be achieved with a low transmission delay of $K$ time slots, which is more bandwidth efficient than the existing protocols. Simulation results also justify the performance gains.
\end{abstract}

\section{INTRODUCTION}

Many-to-one communication, where a set of source nodes send data to a common destination, has many applications in the cellular uplink, sensor network, and surveillance system. As the multiple users need to access the destination simultaneously, the traditional methods try to separate the user signals in the time domain, frequency domain or code domain in order to avoid co-channel interferences. However, the overall system performances may still be unacceptable due to severe channel fading and large path loss.

Cooperative communication can efficiently address the above issue by letting the nodes help each other [1]. Several simple relaying protocols are first proposed in [2], where each node tries to find a neighboring partner to relay its local data. As the destination could receive two independent replicas of the same information, a diversity order of 2 can be achieved to improve transmission reliability. However, two time slots are required to complete the transmission of every source message due to the half-duplex constraints.

Recently, the above single-relay protocols are extended to the multi-node cooperation cases in [3]-[5]. In maximal cooperation protocol [3], all nodes are first ordered according to their distances to the destination. Whenever a node broadcasts its data, all the following nodes would decode-and-forward (DAF) such information in a time-division multiple access (TDMA) way. An incremental diversity is thus achieved with the distant nodes enjoying a higher-order diversity. However, a large delay of $N(N+1) / 2$ time slots is incurred. To overcome such shortcomings, an improved strategy is to allocate a single cooperation phase to each node, and linear coding is used to relay the signals from multiple sources [4]. It has been shown that the same incremental diversity can be achieved with the delay being reduced to $(2 N-1)$ time slots. Yet the best protocol is space-time network coding (STNC) [5], in which the multi-node cooperation is divided into two phases, i.e., a broadcasting phase in which all nodes send out their local data alone, and a cooperation phase in which all nodes relay the data from other sources through linear coding. The transmission in both phases works in a TDMA way to avoid asynchronization problem. Due to symmetry, a diversity order of $N$ is achieved by all nodes with the delay being $2 N$ time slots.

Compared with conventional TDMA protocol, which only introduces a delay of $N$ time slots but provides no spatial diversity, all the above strategies actually sacrifice some extent of spectral efficiency as a tradeoff for spatial diversity. In the applications where the data rate is predetermined, all the nodes have to apply higher order modulations to compensate for the rate loss, which may inversely increase the decoding error even with spatial diversity. Therefore, we develop in this work a new protocol that aims to achieve better tradeoffs between these two performance metrics. The whole network is first divided into several clusters, which send data successively in a TDMA way. Each node would combine its local symbol and relayed symbols from other clusters through linear combining, which are then decoupled using linear decorrelator at the receivers. We show that a diversity order that is close to the number of source nodes can be achieved with the delay being the number of clusters. Therefore, our protocol provides a flexible way to compromise the spectral efficiency and spatial diversity by simply changing the cluster size. Simulation results also validate the performance gains over the existing protocols.

Notations: Boldface uppercase (lowercase) letter represents matrix (vector). $(\cdot)^{*},(\cdot)^{T}$ and $(\cdot)^{H}$ stand for conjugate, transpose and conjugate transpose, respectively. We shall use abbreviation i.i.d. for independent and identically distributed, and denote $Z \sim \mathcal{C N}\left(\mu, \sigma^{2}\right)$ as a circularly symmetric complex Gaussian random variable $Z$ with i.i.d. real part and imaginary part $\sim \mathcal{N}\left(\mu, \frac{\sigma^{2}}{2}\right)$. Finally, the probability of an event $\mathcal{A}$ and the Probability Density Function (PDF) of a continuous random variable $Z$ are denoted by $\operatorname{Pr}(\mathcal{A})$ and $f(Z)$, respectively.

\section{SYSTEM MODEL}

Consider a wireless network with $N$ source nodes sending data to a single destination node $d$ using $M$-ary Phase-Shift Keying (PSK) modulations. All source nodes are divided into $K$ clusters, each having $Q$ nodes, i.e., $N=K Q$. The $i$ th 


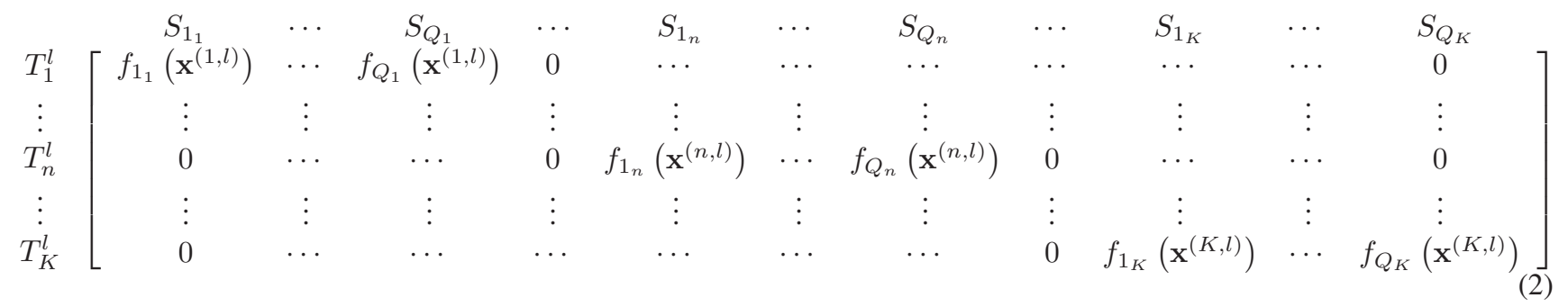

$$
f_{i_{n}}\left(\mathbf{x}^{(n, l)}\right)(t)=\frac{h_{i_{n}, d}^{*}}{\left|h_{i_{n}, d}\right|}\left\{x_{i_{n}}^{l} s_{i_{n}}(t)+\sum_{j=1}^{Q}\left(\sum_{m=1}^{n-1} \sqrt{\mu_{j_{m}, i_{n}} P} I_{j_{m}, i_{n}}^{l} x_{j_{m}}^{l} s_{j_{m}}(t)+\sum_{m=n+1}^{K} \sqrt{\mu_{j_{m}, i_{n}} P} I_{j_{m}, i_{n}}^{l-1} x_{j_{m}}^{l-1} s_{j_{m}}(t)\right)\right\}
$$

$$
y_{i_{n}}^{T_{m}^{l}}(t)=\sum_{j=1}^{Q} h_{j_{m}, i_{n}} f_{j_{m}}\left(\mathbf{x}^{(m, l)}\right)(t)+w_{i_{n}}^{T_{m}^{l}}(t)=\sum_{r=1}^{Q}\left\{\sum_{k=1}^{n} b_{r_{k}, i_{n}}^{(m, l)} x_{r_{k}}^{l} s_{r_{k}}(t)+\sum_{k=n+1}^{K} b_{r_{k}, i_{n}}^{(m,-1)} x_{r_{k}}^{l-1} s_{r_{k}}(t)\right\}+w_{i_{n}}^{T_{m}^{l}}(t)
$$

$$
y_{u_{q}, i_{n}}^{T_{m}^{l}}=\left\langle y_{i_{n}}^{T_{m}^{l}}(t), s_{u_{q}}(t)\right\rangle=\sum_{r=1}^{Q}\left\{\sum_{k=1}^{n} b_{r_{k}, i_{n}}^{(m, l)} x_{r_{k}}^{l} \rho_{r_{k}, u_{q}}+\sum_{k=n+1}^{K} b_{r_{k}, i_{n}}^{(m, l-1)} x_{r_{k}}^{l-1} \rho_{r_{k}, u_{q}}\right\}+w_{u_{q}, i_{n}}^{T_{m}^{l}}
$$

node in the $n$th cluster is denoted by $i_{n}$ for $i=1,2, \cdots, Q$ and $n=1,2, \cdots, K$. Suppose each node has a signature waveform $s_{i_{n}}(t)$, with the cross-correlation being

$$
\begin{aligned}
\rho_{i_{n}, j_{m}} & =\left\langle s_{i_{n}}(t), s_{j_{m}}(t)\right\rangle=\frac{1}{T_{s}} \int_{0}^{T_{s}} s_{i_{n}}(t) s_{j_{m}}^{*}(t) d t \\
& =\left\{\begin{array}{l}
1, \text { if } i=j \text { and } n=m \\
\rho, \text { otherwise }
\end{array}\right.
\end{aligned}
$$

where $T_{s}$ is the symbol period. As [5], we assume these waveforms are known at each node. The storage overhead is not the main concern of this work. The cross-correlation coefficient $\rho \leq 1$ is a design parameter depending on the orthogonality of different waveforms. As will be clear later, $\rho$ only determines the coding gain but is independent of the diversity gain of our protocol.

Without loss of generality, the Additive White Gaussian Noise (AWGN) at any receiver is assumed to be i.i.d. $\mathcal{C N}\left(0, N_{0}\right)$, and the channel between any two nodes $u$ and $v$ is modeled as $h_{u, v} \sim \mathcal{C N}\left(0, \sigma_{u, v}^{2}\right)$. We assume all the channels experience slow fading, and each transmitter knows the phase of its own channel to the destination. When all the nodes operate in the same frequency band and the channels are reciprocal, this can be done by letting the destination broadcast a training sequence such that each node can perform channel estimation and thus acquire the channel phases.

The distributed space-time coding matrix of the proposed protocol is given in (2) on the top of this page, where $l$ is the index of transmission phase. Each transmission phase is divided into $K$ time slots, and all clusters work in a TDMA manner. To be specific, the nodes within the $n$th cluster send its own new packet and help relay the most recent packets of other clusters in the $n$th time $\operatorname{slot}^{1}$, which is denoted by $T_{n}^{l}$. Since the processing on each symbol within a packet is similar, we do not distinguish between the packet and the symbol in the following to simplify the notations. For node $i_{n}$, the transmitted signal is a linear combination of its local symbol $x_{i_{n}}^{l}$, and the relayed symbols sent by other clusters in the previous $K-1$ slots, i.e., $x_{j_{m}}^{l}$ for $j=1,2, \cdots, Q$ and $m=1,2, \cdots, n-1$, and $x_{j_{m}}^{l-1}$ for $j=1,2, \cdots, Q$ and $m=n+1, n+2, \cdots, K$. During the first transmission phase (i.e., $l=1$ ), $x_{j_{m}}^{l-1}$ is set to 0 for $m=n+1, n+2, \cdots, K$ as there are no packets received from these clusters yet. For this cooperation protocol, it is easy to see that there is only intercluster cooperation but no intra-cluster cooperation. So an intuitive clustering strategy is to group the distant nodes in the same cluster while separating the nodes that are geometrically close in different clusters in order to improve the inter-cluster connectivity. However, in a large random network it is almost impossible to fulfill the above two goals at the same time, and random clustering should be a good scheme on average.

Suppose selective DAF protocol [2] is applied by all nodes. The decoding state (i.e., correct decoding or not) is denoted by

$$
I_{j_{m}, i_{n}}^{l}=\left\{\begin{array}{l}
1, \text { if node } i_{n} \text { decode } x_{j_{m}}^{l} \text { correctly } \\
0, \text { otherwise }
\end{array}\right.
$$

for $m \neq n$. We further define

$$
I_{j_{m}, i_{n}}^{l}=\left\{\begin{array}{l}
1, j=i \\
0, \text { otherwise }
\end{array}\right.
$$

for $m=n$ for notational convenience. As [2]-[5], we assume the decoding state can be perfectly detected via certain er-

\footnotetext{
${ }^{1}$ We assume the transmitters are perfectly synchronized. The effect of synchronization errors is beyond the scope of this work.
} 
ror detection technique like cyclic redundancy check or by comparing the instantaneous received Signal-To-Noise Ratio (SNR) with a predefined threshold. Then the symbol $x_{j_{m}}^{l}$ $\left(x_{j_{m}}^{l-1}\right)$ will be relayed by node $i_{n}$ only if $I_{j_{m}, i_{n}}^{l}=1$ $\left(I_{j_{m}, i_{n}}^{l-1}=1\right)$ for $m \neq n$. Let $\mathbf{x}_{k}^{l}=\left(x_{1_{k}}^{l}, x_{2_{k}}^{l}, \cdots, x_{Q_{k}}^{l}\right)^{T}$ be the local symbol vector generated by the $k$ th cluster in the $l$ th transmission phase, then the super symbol vector transmitted in the time slot $T_{n}^{l}$ is $\mathbf{x}^{(n, l)}=$ $\left(\mathbf{x}_{1}^{l^{T}}, \cdots, \mathbf{x}_{n}^{l}{ }^{T}, \mathbf{x}_{n+1}^{l-1}{ }^{T}, \cdots, \mathbf{x}_{K}^{l-1^{T}}\right)^{T}$. With the above notations, the transmitted signal of node $i_{n}$ is given by (5) on the top of previous page, where $P$ is the total transmitted power, $\mu_{j_{m}, i_{n}}$ is the portion of power used to send node $j_{m}$ 's message. The beamforming coefficient $\frac{h_{i_{n}, d}^{*}}{\left|h_{i_{n}, d}\right|}$ depends only on the channel phase of $h_{i_{n}, d}$, and is such to make sure that the same relayed symbol transmitted by different nodes are combined coherently at the destination.

\section{LINEAR MULTIUSER DETECTION}

In this section, we shall develop the signal model when linear decorrelator [6] is used at both the source nodes and destination to separate the source signals. Perfect synchronization among the nodes within the same cluster is assumed to simplify the analysis. The derived Symbol Error Rate (SER) can thus be regarded as a low bound for the practical system with timing mismatch.

\section{A. Source Decoding}

With the transmitted signal in (5), the received signal during time slot $T_{m}^{l}$ at the source node $i_{n}$ for $n \neq m$ is given by (6) on the top of previous page, where

$$
b_{r_{k}, i_{n}}^{(m, l)}=\sum_{j=1}^{Q} h_{j_{m}, i_{n}} \frac{h_{j_{m}, d}^{*}}{\left|h_{j_{m}, d}\right|} \sqrt{\mu_{r_{k}, j_{m}} P} I_{r_{k}, j_{m}}^{l}
$$

is the equivalent channel coefficient from the $m$ th cluster to node $i_{n}$ for symbol $x_{r_{k}}^{l}$. Although the transmitted symbols are mixed with each other in the air, a linear decorrelator can be used to decouple these symbols. The received signal $y_{i_{n}}^{T_{m}^{l}}(t)$ is first fed into the matched filter bank $\left\{s_{u_{q}}(t)\right\}$ for $u=$ $1,2, \cdots, Q$ and $q=1,2, \cdots, K$ to obtain (8) shown on the top of previous page. These scalars can further be put together in a more compact matrix form as

$$
\mathbf{y}_{i_{n}}^{T_{m}^{l}}=\left(y_{1_{1}, i_{n}}^{T_{m}^{l}}, \cdots, y_{Q_{K}, i_{n}}^{T_{m}^{l}}\right)^{T}=\mathbf{R B}_{i_{n}}^{(m, l)} \mathbf{x}^{(m, l)}+\mathbf{w}_{i_{n}}^{T_{m}^{l}},
$$

where $\mathbf{R}$ is the correlation matrix of the signature waveforms with 1 on the diagonal and all the off-diagonal elements being $\rho, \mathbf{B}_{i_{n}}^{(m, l)}=\operatorname{diag}\left(b_{1_{1}, i_{n}}^{(m, l)}, \cdots, b_{Q_{m}, i_{n}}^{(m, l)}, b_{1_{m+1}, i_{n}}^{(m, l-1)}, \cdots, b_{Q_{K}, i_{n}}^{(m, l-1)}\right)$ is a diagonal matrix with the equivalent channel coefficients for the corresponding symbols on the main diagonal, and $\mathbf{w}_{i_{n}}^{T_{m}^{l}} \sim \mathcal{C N}\left(0, N_{0} \mathbf{R}\right)$ is the equivalent AWGN vector. Now the source symbols can be easily decoupled by pre-multiplying $\mathbf{y}_{i_{n}}^{T_{m}^{l}}$ with $\mathbf{R}^{-1}$, i.e.,

$$
\tilde{\mathbf{y}}_{i_{n}}^{T_{m}^{l}}=\mathbf{R}^{-1} \mathbf{y}_{i_{n}}^{T_{m}^{l}}=\mathbf{B}_{i_{n}}^{(m, l)} \mathbf{x}^{(m, l)}+\tilde{\mathbf{w}}_{i_{n}}^{T_{m}^{l}} .
$$

After the source signals are separated, the element-wise single symbol decoding can be performed on

$$
\begin{aligned}
\tilde{y}_{j_{m}, i_{n}}^{T_{m}^{l}} & =b_{j_{m}, i_{n}}^{(m, l)} x_{j_{m}}^{l}+\tilde{w}_{j_{m}, i_{n}}^{T_{m}^{l}} \\
& =h_{j_{m}, i_{n}} \frac{h_{j_{m}, d}^{*}}{\left|h_{j_{m}, d}\right|} \sqrt{\mu_{j_{m}, j_{m}} P} x_{j_{m}}^{l}+\tilde{w}_{j_{m}, i_{n}}^{T_{m}^{l}}
\end{aligned}
$$

to extract the local symbol $x_{j_{m}}^{l}$ for $j=1,2, \cdots, Q$ transmitted by the $m$ th cluster during $T_{m}^{l}$, where $\tilde{w}_{j_{m}, i_{n}}^{T_{m}^{l}} \sim \mathcal{C N}\left(0, N_{0} \rho_{N}\right)$ is the equivalent AWGN and

$$
\rho_{N}=\frac{1+(N-2) \rho}{1+(N-2) \rho-(N-1) \rho^{2}}
$$

is the noise enhancement factor due to decorrelation. The conditional SNR is given by

$$
\gamma_{j_{m}, i_{n} \mid h_{j_{m}, i_{n}}}^{l}=\frac{\mu_{j_{m}, j_{m}} \gamma}{\rho_{N}}\left|h_{j_{m}, i_{n}}\right|^{2},
$$

where $\gamma=\frac{P}{N_{0}}$ is defined as the system SNR.

\section{B. Destination Decoding}

The source signals can be decoupled in a similar way at the destination. The vector output during time slot $T_{m}^{l}$ is given by

$$
\tilde{\mathbf{y}}_{d}^{T_{m}^{l}}=\mathbf{A}_{d}^{(m, l)} \mathbf{x}^{(m, l)}+\tilde{\mathbf{w}}_{d}^{T_{m}^{l}}
$$

where $\tilde{\mathbf{w}}_{d}^{T_{m}^{l}} \sim \mathcal{C N}\left(\mathbf{0}, N_{0} \mathbf{R}^{-1}\right)$ is the equivalent AWGN, and $\mathbf{A}_{d}^{(m, l)}=\operatorname{diag}\left(a_{1_{1}, d}^{(m, l)}, \cdots, a_{Q_{m}, d}^{(m, l)}, a_{1_{m+1}, d}^{(m, l-1)}, \cdots, a_{Q_{K}, d}^{(m, l-1)}\right)$ is a diagonal matrix with the equivalent channel coefficients

$$
a_{i_{n}, d}^{(m, l)}=\sum_{j=1}^{Q}\left|h_{j_{m}, d}\right| \sqrt{\mu_{i_{n}, j_{m}} P} I_{i_{n}, j_{m}}^{l}
$$

from the $m$ th cluster to the destination on the main diagonal. It is easy to see that the signals from different nodes are combined coherently in the air due to transmit beamforming. Besides, as the destination can obtain a set of $K$ replicas for any source symbol $x_{j_{m}}^{l}$ in the consecutive time slots $T_{m}^{l}, \cdots, T_{m-1}^{l+1}$, the decoding error can be further reduced through Equal Gain Combining (EGC), i.e.,

$$
\tilde{x}_{j_{m}, d}^{l}=\sum_{n=m}^{K} \tilde{y}_{j_{m}, d}^{T_{n}^{l}}+\sum_{n=1}^{m-1} \tilde{y}_{j_{m}, d}^{T_{n}^{l+1}}=\tilde{h}_{j_{m}, d}^{l} x_{j_{m}}^{l}+\tilde{w}_{j_{m}, d}^{l},
$$

where $\tilde{h}_{j_{m}, d}^{l}=\sum_{i_{n} \in \epsilon_{1} \psi_{j_{m}}^{l} \cup\left\{j_{m}\right\}}\left|h_{i_{n}, d}\right| \sqrt{\mu_{j_{m}, i_{n}}}$ is the equivalent channel with ${ }_{1} \psi_{j_{m}}^{l}=\left\{i_{n}: I_{j_{m}, i_{n}}^{l}=1, n \neq m\right\}$ being the set of source nodes that can decode $x_{j_{m}}^{l}$ correctly, and $\tilde{w}_{j_{m}, d}^{l} \sim$ $\mathcal{C N}\left(0, N_{0} \rho_{N} K\right)$ is the equivalent AWGN. As the equivalent channel $\tilde{h}_{j_{m}, d}^{l}$ is a function of both the decoding states and real channel coefficients, the conditional SNR is then

$$
\gamma_{j_{m} \mid\{h\},\left\{I_{j_{m}, i_{n}}^{l}\right\}}^{l}=\frac{\gamma}{\rho_{N} K}\left|\tilde{h}_{j_{m}, d}^{l}\right|^{2} .
$$

It is worth noting that some soft symbols $\tilde{y}_{j_{m}, d}^{T_{n}^{l}}\left(\tilde{y}_{j_{m}, d}^{T_{n}^{l+1}}\right)$ may be pure noise. This may occur when no nodes in the $n$th cluster can decode $x_{j_{m}}^{l}$ correctly. So it seems better to exclude 
such soft symbols during EGC to suppress the noise power. However, this requires the destination to know the decoding states at all source nodes, which incurs a tremendous amount of feedback overhead. So in this work, we assume the decoding states are only local information, and the destination would combine all the soft symbols no matter they contain the source information or not.

\section{A. Exact SER Analysis}

\section{PERFORMANCE ANALYSIS}

Given the channel coefficient, the conditional SER for the $M$-ary PSK signal is [7]

$$
\varphi\left(\gamma_{\mid\{h\}}\right)=\frac{1}{\pi} \int_{0}^{\frac{M-1}{M} \pi} \exp \left(-\frac{g_{p s k} \gamma_{\mid\{h\}}}{\sin ^{2} \theta}\right) d \theta,
$$

where $g_{p s k}=\sin ^{2}\left(\frac{\pi}{M}\right)$ is a constant determined by the constellation size $M$. The unconditional SER can then be obtained after averaging the above expression over channel distribution.

However, the SER analysis for the proposed protocol is complicated as the SNR expression (17) also depends on the decoding states at other clusters. To facilitate the analysis, define the decoding states vector for symbol $x_{j_{m}}^{l}$ as $\mathbf{I}_{j_{m}}^{l}=\left(\mathbf{I}_{j_{m}, 1}^{l}, \cdots, \mathbf{I}_{j_{m}, m-1}^{l}, \mathbf{I}_{j_{m}, m+1}^{l}, \cdots, \mathbf{I}_{j_{m}, K}^{l}\right)$ with $\mathbf{I}_{j_{m}, n}^{l}=\left(I_{j_{m}, 1_{n}}^{l}, I_{j_{m}, 2_{n}}^{l}, \cdots, I_{j_{m}, Q_{n}}^{l}\right)$. Note that $\mathbf{I}_{j_{m}}^{l}$ is actually a random binary vector of length $Q(K-1)$, so it can also be represented by the corresponding baseten number for notational convenience, i.e., $\left|\mathbf{I}_{j_{m}, n}^{l}\right|_{2}=$ $\left[\mathbf{I}_{j_{m}, 1}^{l}, \cdots, \mathbf{I}_{j_{m}, m-1}^{l}, \mathbf{I}_{j_{m}, m+1}^{l}, \cdots, \mathbf{I}_{j_{m}, K}^{l}\right]_{2}$. Besides, as the decoding states at different nodes are independent, all the elements of the vector $\mathbf{I}_{j_{m}}^{l}$ are thus independent Bernoulli random variables with $\mathrm{PDF}$

$$
\operatorname{Pr}\left(I_{j_{m}, i_{n}}^{l}\right)=P_{j_{m}, i_{n}}^{1-I_{j_{m}, i_{n}}^{l}}\left(1-P_{j_{m}, i_{n}}\right)^{I_{j_{m}, i_{n}}^{l}} .
$$

The PDF for the decoding states vector $\mathbf{I}_{j_{m}}^{l}$ is then

$$
\begin{aligned}
\operatorname{Pr}\left(\mathbf{I}_{j_{m}}^{l}\right) & =\prod_{i=1}^{Q} \prod_{\substack{n=1 \\
n \neq m}}^{K} \operatorname{Pr}\left(I_{j_{m}, i_{n}}^{l}\right) \\
& =\prod_{i_{n} \in \psi_{0} \psi_{j_{m}}^{l}} P_{j_{m}, i_{n}} \prod_{i_{n} \in \psi_{1} \psi_{j_{m}}^{l}}\left(1-P_{j_{m}, i_{n}}\right),
\end{aligned}
$$

where ${ }_{0} \psi_{j_{m}}^{l}=\left\{i_{n}: I_{j_{m}, i_{n}}^{l}=0, n \neq m\right\}$ is the set of source nodes that fail to decode $x_{j_{m}}^{l}$ correctly, and

$$
\begin{aligned}
P_{j_{m}, i_{n}} & =E_{h_{j_{m}, i_{n}}} \varphi\left(\frac{\mu_{j_{m}, j_{m}} \gamma}{\rho_{N}}\left|h_{j_{m}, i_{n}}\right|^{2}\right) \\
& =\frac{1}{\pi} \int_{0}^{\frac{M-1}{M} \pi}\left(1+\frac{\mu_{j_{m}, j_{m}} \sigma_{j_{m}, i_{n}}^{2} g_{p s k} \gamma}{\rho_{N} \sin ^{2} \theta}\right)^{-1} d \theta
\end{aligned}
$$

is the decoding error for symbol $x_{j_{m}}^{l}$ at the source node $i_{n}$.

Now according to the law of total probability, the decoding error for symbol $x_{j_{m}}^{l}$ at the destination is

$$
P_{e}\left(x_{j_{m}}^{l}\right)=\sum_{\left|\mathbf{I}_{j_{m}}^{l}\right|_{2}=0}^{2^{Q(K-1)}-1} P_{e}\left(x_{j_{m}}^{l} \mid \mathbf{I}_{j_{m}}^{l}\right) \operatorname{Pr}\left(\mathbf{I}_{j_{m}}^{l}\right),
$$

where $P_{e}\left(x_{j_{m}}^{l} \mid \mathbf{I}_{j_{m}}^{l}\right)$ is the conditional SER given the decoding states. If $\left|{ }_{1} \psi_{j_{m}}^{l}\right|=0$, i.e., no source nodes are able to decode symbol $x_{j_{m}}^{l_{m}}$, then the decoding is based totally on the received signal from the direct link, and it is easy to show

$$
\begin{aligned}
& P_{e}\left(\left.x_{j_{m}}^{l}|| \mathbf{I}_{j_{m}}^{l}\right|_{2}=0\right) \\
& \quad=\frac{1}{\pi} \int_{0}^{\frac{M-1}{M} \pi}\left(1+\frac{\mu_{j_{m}, j_{m}} \sigma_{j_{m}, d}^{2} g_{p s k} \gamma}{\rho_{N} K \sin ^{2} \theta}\right)^{-1} d \theta .
\end{aligned}
$$

On the other hand, when $\left|{ }_{1} \psi_{j_{m}}^{l}\right|>0$ we actually have to evaluate the SER of PSK signal using EGC with $\left(\left|{ }_{1} \psi_{j_{m}}^{l}\right|+1\right)$ branches. Unfortunately, no exact closed-form expression has been found in the past decades except for the special case with two branches. Therefore, we apply the Gauss-Hermite quadrature approximation developed in [8], which is given by (24) shown on the top of next page with the integrand given in (25). $z_{k}$ are the zeros of the $N_{p}$-th order Hermite polynomial, and $H_{z_{k}}$ are the weights tabulated in [9, p. 924, table (25.10)]. It has been shown that $N_{p}=20$ is enough to accurately characterize the SER greater than $10^{-5}$. The other functions in (25) are defined respectively as

$$
\begin{aligned}
& \eta_{j_{m}}^{l}(\theta)=\frac{\sin ^{2} \theta}{2 A_{p s k}^{2}}+\frac{1}{4} \sum_{i_{n} \in_{1} \psi_{j_{m}}^{l} \cup\left\{j_{m}\right\}} \mu_{j_{m}, i_{n}} \sigma_{i_{n}, d}^{2}, \\
& X(\theta)=\sqrt{\frac{\pi}{2} \frac{\sin \theta}{A_{p s k}}} \\
& Y(\nu, \theta)=-\frac{\nu \sin ^{2} \theta}{A_{p s k}^{2}}{ }_{1} F_{1}\left(\frac{1}{2} ; \frac{3}{2} ; \frac{\nu^{2} \sin ^{2} \theta}{2 A_{p s k}^{2}}\right) \\
& A_{j_{m}, i_{n}}(\nu)={ }_{1} F_{1}\left(-\frac{1}{2} ; \frac{1}{2} ; \frac{\nu^{2} \mu_{j_{m}, i_{n}} \sigma_{i_{n}, d}^{2}}{4}\right) \\
& B_{j_{m}, i_{n}}(\nu)=\Gamma\left(\frac{3}{2}\right) \nu \sqrt{\mu_{j_{m}, i_{n}} \sigma_{i_{n}, d}^{2}},
\end{aligned}
$$

where ${ }_{1} F_{1}(\cdot ; \cdot ; \cdot)$ is the Kummer confluent hypergeometric function, $\Gamma(\cdot)$ is the gamma function, and $A_{p s k}=\sqrt{\frac{2 \gamma g_{p s k}}{K \rho_{N}}}$ is a constant. Finally, plugging (20), (23) and (24) back into (22) leads to the closed-form decoding error. As will be shown in the next section, the above result matches well with the simulations.

\section{B. Asymptotic SER Analysis}

To gain more insights into the benefits of multi-node cooperation, we shall derive in this subsection the asymptotic SER in the high SNR regions, i.e., when $\gamma \gg 1$. It is easy to check that the source decoding error $P_{j_{m}, i_{n}}$ in (21) would be approaching zero, so we can approximate (20) as

$$
\operatorname{Pr}\left(\mathbf{I}_{j_{m}}^{l}\right) \approx \prod_{i_{n} \in \psi_{0} \psi_{j_{m}}^{l}} P_{j_{m}, i_{n}} .
$$

After plugging the above expression back into (22), we would arrive at (32) shown on the top of next page. A direct observation is that all terms within the summation actually have the similar form of $E_{\tilde{h}} \varphi\left(c \gamma \tilde{h}^{2}\right)$, where $c$ is a constant and 


$$
\begin{aligned}
& P_{e}\left(x_{j_{m}}^{l}|| \mathbf{I}_{j_{m}}^{l} \mid>0\right) \approx \frac{1}{2 \pi^{2}} \int_{0}^{\frac{M-1}{M} \pi} \frac{1}{\sqrt{\eta_{j_{m}}^{l}(\theta)}} \sum_{k=1}^{N_{p}} H_{z_{k}} F_{j_{m}}^{l}\left(\frac{z_{k}}{\sqrt{\eta_{j_{m}}^{l}(\theta)}}, \theta\right) d \theta \\
& F_{j_{m}}^{l}(\nu, \theta)=\operatorname{Re}\left\{(X(\theta)+j Y(\nu, \theta)) \prod_{i_{n} \in \psi_{1} \psi_{j_{m}}^{l} \cup\left\{j_{m}\right\}}\left(A_{j_{m}, i_{n}}(\nu)+j B_{j_{m}, i_{n}}(\nu)\right)\right\}
\end{aligned}
$$

$$
P_{e}\left(x_{j_{m}}^{l}\right) \approx \sum_{\left|\mathbf{I}_{j_{m}}^{l}\right|_{2}=0}^{2^{Q(K-1)}-1} E_{\{h\}} \varphi\left(\frac{\gamma}{\rho_{N} K}\left|\sum_{i_{n} \in \psi_{1} \psi_{m}^{l} \cup\left\{j_{m}\right\}}\right| h_{i_{n}, d}\left|\sqrt{\mu_{j_{m}, i_{n}}}\right|^{2}\right) \prod_{i_{n} \in \psi_{0} \psi_{j_{m}}^{l}} E_{\{h\}} \varphi\left(\frac{\mu_{j_{m}, j_{m}} \gamma}{\rho_{N}}\left|h_{j_{m}, i_{n}}\right|^{2}\right)
$$

$$
P_{e}\left(x_{j_{m}}^{l}\right) \approx\left(\frac{\rho_{N}}{g_{p s k} \gamma}\right)^{N-Q+1} \sum_{\left|\mathbf{I}_{j_{m}}^{l}\right|_{2}=0}^{2^{Q(K-1)}} \frac{K^{\left|{ }_{1} \psi_{j_{m}}^{l}\right|+1} \Gamma\left(\left|{ }_{1} \psi_{j_{m}}^{l}\right|+1\right) 2^{\mid{ }_{1} \psi_{j_{m}}^{l}} \mid G\left(M,\left.\right|_{1} \psi_{j_{m}}^{l} \mid+1\right)(G(M, 1))^{\left|0 \psi_{j_{m}}^{l}\right|}}{\Gamma\left(2\left(\left.\right|_{1} \psi_{j_{m}}^{l} \mid+1\right)\right) \prod_{i_{n} \in \in_{0} \psi_{j_{m}}^{l}} \mu_{j_{m}, j_{m}} \sigma_{j_{m}, i_{n}}^{2} \prod_{i_{n} \in \epsilon_{1} \psi_{j_{m}}^{l} \cup\left\{j_{m}\right\}} \mu_{j_{m}, i_{n}} \sigma_{i_{n}, d}^{2}}
$$

$\tilde{h}=\sum_{i=1}^{L}\left|h_{i}\right|$ is the equivalent channel with $h_{i} \sim \mathcal{C N}\left(0, \sigma_{i}^{2}\right)$ being independent random variables. It has been proved in [10] that at high SNRs, this metric depends only on the behavior of the distribution of $\tilde{h}$ around the origin. Using Taylor series expansion, we can obtain

$$
f_{\tilde{h}}(x) \approx \frac{2^{L}}{\Gamma(2 L) \prod_{l=1}^{L} \sigma_{l}^{2}} x^{2 L-1}+o\left(x^{2 L}\right) .
$$

With the above result, it is easy to show that

$$
E_{\tilde{h}} \varphi\left(c \gamma \tilde{h}^{2}\right) \approx \frac{\Gamma(L) 2^{L-1}}{\Gamma(2 L)\left(g_{p s k} c \gamma\right)^{L} \prod_{l=1}^{L} \sigma_{l}^{2}} G(M, L),
$$

where

$$
G(M, L)=\frac{1}{\pi} \int_{0}^{\frac{M-1}{M} \pi} \sin ^{2 L} \theta d \theta
$$

is a constant depending on the constellation size $M$ and the number of combining branches $L$. Finally, plugging (34) back into (32) and doing some manipulation leads to the asymptotic SER (36) shown on the top of this page.

The diversity order is defined as

$$
\operatorname{div}=-\lim _{\bar{\gamma} \rightarrow \infty} \frac{\log P_{e}(\bar{\gamma})}{\log \bar{\gamma}}
$$

From (36), it is easy to see that a diversity order of $(N-Q+1)$ can be achieved, and the transmission delay is $K=N / Q$ time slots as the destination has to collect $K$ replicas to perform EGC. For the special case without clustering, i.e., $Q=1$ and $K=N$, it achieves a diversity order of $N$ at a cost of longer delay of $N$ time slots. Therefore, the total delay is inversely proportional to the cluster size $Q$ while the diversity order is only decreasing linearly with $Q$. When the network size $N \gg 1$, the proposed scheme actually provides an efficient way to significantly improve the spectral efficiency at a trivial loss of diversity order by properly choosing the cluster size. It is also observed that the diversity gain is independent of the cross correlation factor $\rho$. However, as $\rho_{N}$ is an increasing function of $\rho$, the coding gain is directly determined by the orthogonality of the signature waveforms.

\section{SIMULATIONS}

In the following, we shall present some simulation results to study the performances of the proposed scheme. All channels are independent, and the channel gain is modeled as $\sigma^{2}=d^{-3}$, where $d$ is the distance between the associated two nodes. In all cases, the transmitted power is equally allocated to transmit the local symbol and relayed symbols.

The SER performances with and without clustering are given in Fig. 1. The four nodes are symmetrically located on a unit circle with the destination being at the center. For the case $K=2$, the two nodes on the same diameter are clustered together, and QPSK signals are used by all nodes. Clearly, the simulation results match well with the theoretical analysis (22) when the SER is greater than $10^{-5}$. When the SER is low, the Gauss-Hermit quadrature is not a good approximation, but the asymptotic analysis (36) is tight since the SNR is high in such cases. It is also observed that the curve corresponding to $K=4$ clusters has steeper slope than that having $K=2$ clusters in the high SNR regions. This is consistent with our analysis, as the former has a diversity order of 4 while the latter only has a diversity order of 3 . Another observation is that when the cross correlation factor $\rho$ becomes larger, the proposed scheme would suffer some loss in coding gain, though the diversity order is still the same. This is because the noise enhancement factor $\rho_{N}$ in (12) is actually an increasing function of $\rho$. So the equivalent noise power at the decorrelator output will increase accordingly.

To gain more insights into the advantage of the proposed protocol, we also compare the performances with conventional TDMA and STNC protocol [5] after carefully normalizing the rate and power. The four source nodes are now randomly 


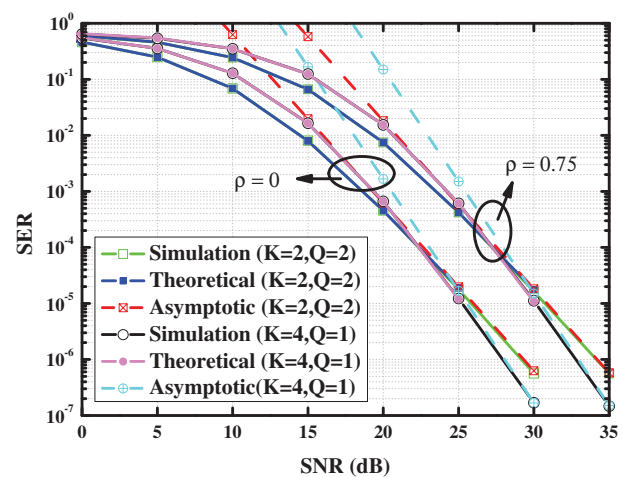

Fig. 1. SER performances with QPSK modulations.

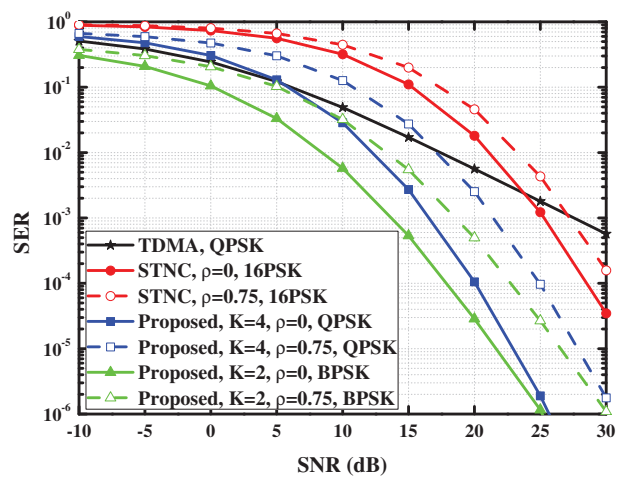

Fig. 2. SER comparison in a $2 \times 2$ network.

generated on a square with the destination being at the center. We show the results in Fig. 2 and Fig. 3 when the square size is $2 \times 2$ and $4 \times 4$, respectively. In the case of clustering (i.e., $K=2$ ), the two source nodes having the largest distance are always grouped together to improve inter-cluster connectivity. It is observed that the proposed scheme has a huge performance gain. Compared with STNC, which also achieves a diversity order of 4 as the proposed scheme with $K=4$ clusters, the coding gain is about $8 \mathrm{~dB}$ in the high SNR regions in Fig. 2. This is because the STNC scheme is not bandwidth efficient due to the separation of broadcasting phase and cooperation phase, whereas in our scheme these two phases have been combined through smart design of the space-time codes. It is also observed that both of STNC and the proposed scheme with $K=4$ perform worse than TDMA in the low SNR regions in Fig. 2. This is because for the nodes located far away from each other, the cooperation is not effective due to higher decoding error. So part of the transmitted power reserved to relay symbols is actually wasted in most cases. Another interesting observation is that the proposed scheme with $K=2$ actually performs best in all cases, though the diversity order is only 3 . The reason is that the nodes having large distance are always grouped in the same cluster, so the inter-cluster communications are really reliable. Fig. 3 shows the throughput in a $4 \times 4$ network, which is defined as the number of bits per channel use (bpcu) that

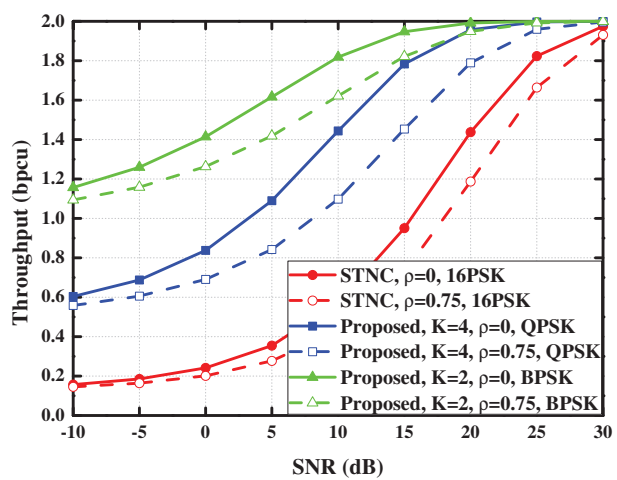

Fig. 3. Throughput comparison in a $4 \times 4$ network.

can be successfully delivered to the destination. The simulation results confirm again the huge throughput gain of our scheme due to more efficient use of channel resources. Comparatively, the throughput gain is more eminent in the low-to-medium SNR regions, in which case the user cooperation is not fully effective and thus to improve the spectral efficiency is more important to achieve a better overall performance.

\section{CONCLUSIONS AND FUTURE WORK}

We proposed a novel clustering based space-time network coding protocol to achieve spatial diversity in a many-to-one communication scenario. Both of the exact and asymptotic SER expressions were derived and it was shown that a diversity order of $(N-Q+1)$ can be achieved with a low delay of $K$ time slots. The high spectral efficiency leads to significant performance gain over the existing STNC scheme. Future work may concern the asynchronization problem within the cluster. One may also develop the clustering algorithm and address the rate and power allocation issues.

\section{REFERENCES}

[1] K. J. R. Liu, A. K. Sadek, W. Su, and A. Kwasinski, Cooperative Communications and Networking, Cambridge University Press, 2008

[2] J. N. Laneman, D. N. C. Tse, and G. W. Wornell, "Cooperative Diversity in Wireless Networks: Efficient Protocols and Outage Behavior," IEEE Trans. Inf. Theory, vol. 50, no. 12, pp. 3062-3080, Dec. 2004.

[3] A. K. Sadek, W. Su, and K. J. R. Liu, "Multinode Cooperative Communications in Wireless Networks," IEEE Trans. Signal Process., vol. 55, no. 1, pp. 341-355, Jan. 2007.

[4] H. Q. Lai, A. S. Ibrahim, and K. J. R. Liu, "Wireless Network Cocast: Location-Aware Cooperative Communications using Network Coding," IEEE Trans. on Wireless Commun., vol. 8, no. 7, pp. 3844-3854, July 2009.

[5] H. Q. Lai and K. J. R. Liu, "Space-Time Network Coding," IEEE Trans. on Signal Process., vol. 59, no. 4, pp. 1706-1718, Apr. 2011.

[6] S. Verdu, Multiuser Detection. Cambridge University Press, 1998.

[7] M. K. Simon and M. S. Alouini, "A unified approach to the performance analysis of digital communications over generalized fading channels," Proc. IEEE, vol. 86, no. 9, pp. 1860-1877, Sep. 1998.

[8] M. S. Alouini and M. K. Simon, "Performance analysis of coherent equal gain combiing over nakami-m fading channels," IEEE Trans. on Vehicular Technology, vol. 50, pp. 1449-1462, Dec. 2001.

[9] M. Abramowitz and I. A. Stegun, Handbook of Mathematical Functions, with Formulas, Graphs and Mathematical Tables. New York: Dover publications, 9th Edition, 1970.

[10] Z. Wang and G. B. Giannakis, "A simple and general parameterization quantifying performance in fading channels," IEEE Trans. Commun., vol. 51, no. 8, pp. 1389-1398, Aug. 2003. 